\title{
Estrategias de \\ enseñanza y \\ aprendizaje en \\ el bachillerato \\ mixto del \\ CECAD UABJO
}

Teresa Guadalupe Antonio-Cruz 


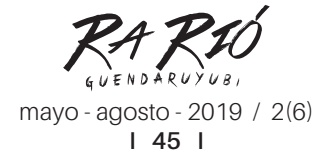

I 45 I

\section{Resumen}

Todo proceso formativo engloba la toma de decisiones y un conjunto de relaciones entre diversos actores, cuya prioridad es generar acciones que permitan la construcción de saberes y conocimientos. En este objetivo tienen lugar especial las propuestas educativas y modalidades de estudio desde las cuales se desarrolla la formación de estudiantes. Asimismo, hablar de enseñanza y aprendizaje en educación media superior es tomar en cuenta las características propias del tipo educativo y la diversidad de opciones y estrategias para realizarlos. En este artículo se enuncian los rasgos del bachillerato mixto que oferta el Centro de Educación Continua Abierta y a Distancia de la Universidad Autónoma "Benito Juárez" de Oaxaca (CECAD-UABJO), a fin de dar cuenta cómo se lleva a cabo la formación en la modalidad mixta y las estrategias diseñadas para su implementación en el programa educativo de bachillerato.

Palabras clave: bachillerato mixto, estrategias de aprendizaje, estrategias de enseñanza, modalidad mixta.

\section{Abstract}

Every educational process involves making decisions and a set of relations between diverse actors whose priority is to carry out educational activities that will allow the acquisition of knowledge. To reach this goal, educational projects and study fashions that develop a student's formation hold a special place. Likewise, to talk about teaching and learning at a high school level is to notice the characteristics of each educational type, as well as the diversity of options and strategies to guide teaching and learning. This paper enunciates the distinctive traits of the mixed high school at the Center for Continuous Open and Long-Distance Education at the Benito Juarez Autonomous University of Oaxaca (CECAD-UABJO), in order to point out how learning is carried out in this mixed mode, as well as the strategies designed to be implemented at a high school program.

Keywords: mixed high school, learning strategies, teaching strategies, mixed modes. 


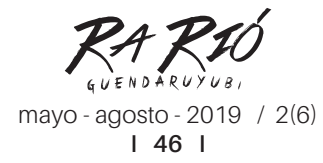

La modalidad mixta, dada su característica particular de integrar la instrucción presencial y a distancia, representa una opción necesaria para ampliar el número de bachilleratos a gran escala. Actualmente, diversos estudios demuestran que las y los jóvenes de nuestras sociedades pasan más horas frente a una computadora o a dispositivos tecnológicos, conectados e interactuando desde diversos lugares y con distintos fines, lo cual impacta directamente en su formación.

Esta modalidad no se entiende únicamente como un aprendizaje que se complementa con el uso de recursos o materiales diseñados para trabajar a distancia o virtuales, ni como el uso de tecnologías, medios y recursos tecnológicos para el desarrollo de contenidos, sino que se fortalece, además, con la interacción directa, cara a cara entre docenteestudiante y estudiante-estudiante. Este es el reto de la modalidad mixta, no sólo consiste en combinar procesos o tipos de instrucciones, sino en identificar las necesidades reales de los y las estudiantes que optan por esta alternativa y definir un modelo flexible, innovador y pertinente.

La opción mixta es conocida también con el término anglosajón blended learning o $B$-Learning, que es un modelo utilizado para diseñar ambientes educativos mixtos. El término tiene tendencia con una marcada raíz procedente del campo de la Psicología escolar, en la que destaca la palabra "aprendizaje" como contrapuesta a "enseñanza", con la finalidad de enfatizar el papel del estudiante y que la enseñanza se centre en él (Bartolomé-Pina, 2008 en Silva, Mendoza \& Guarneros, s.f., p. 2). 

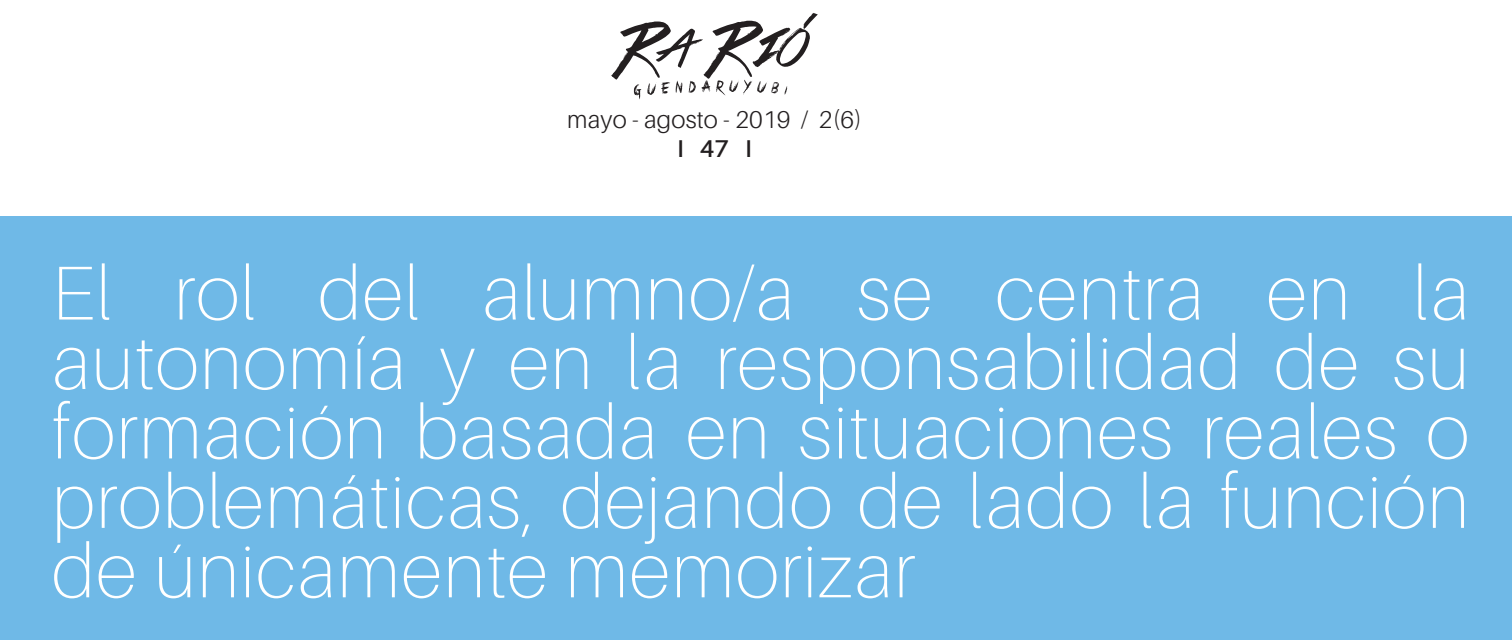

De acuerdo con Silva, Mendoza y Guarneros (s.f.), el hecho de que se combinen dos modelos de enseñanza y aprendizaje históricamente diferenciados por sus fines y alcances identifica a la modalidad como mixta. En síntesis, la definen como:

producto de la convergencia de dos paradigmas, en estricto sentido kuhnianos, de ambientes de aprendizaje. Por un lado, está el ambiente de aprendizaje tradicional presencial que ha existido a lo largo de los siglos. Por otro lado, se tiene un ambiente de aprendizaje distribuido que ha comenzado a crecer y expandirse exponencialmente de distintos modos a través del uso de las tecnologías de la información y comunicación, las cuales han ampliado no solamente las posibilidades de distribución de información sino también la interacción humana (Silva, Mendoza \& Guarneros, s.f., p. 3).

El enfoque que se retoma para el bachillerato mixto respecto del proceso de formación de las/los estudiantes tiene su fundamento psicopedagógico en el paradigma centrado en el aprendizaje, el cual proviene del constructivismo, perspectiva epistemológica y psicológica que explica la manera en que conocemos, descrito como "la posibilidad de que el ser humano se vea implicado en la construcción de sus propios conocimientos, partiendo de lo que posee en la estructura cognitiva enriquecida a lo largo de toda la vida y en el intercambio con otros" (Pimienta, 2012, p. 11).

En el modelo centrado en el aprendizaje se reorienta el papel del/la docente y el/la estudiante. La nueva actuación del profesor o profesora contemplael papel de orientador, facilitadory guía delaprendizaje. Porotra parte, el rol del alumno/a se centra en la autonomía y en la responsabilidad de su formación basada en situaciones reales o problemáticas, dejando de lado la función de únicamente memorizar (Ruiz, 2012).

En resumen, se consideran tres elementos fundamentales para generar los ambientes de educación mixta: 


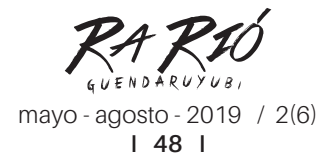

148 I

1. Diversidad de oportunidades para exponer los recursos de aprendizaje y las vías de comunicación entre el profesor/aestudiante, será presencial y a través de medios virtuales

2. La/el estudiante es una persona activa en su propio proceso formativo, así como en la selección de los recursos didácticos pertinentes para su situación individual

3. El diseño de recursos, materiales y contenidos propuestos por la/ el docente.

Las estrategias de aprendizaje son una guía flexible y consciente para alcanzar el logro de objetivos propuestos para el proceso de aprendizaje. Como guía debe contar con pasos definidos, teniendo en cuenta la naturaleza de la estrategia (UNED, s.f., p. 3).

En un modelo de educación mixta la actuación del docente que está en contacto con los alumnos se valdrá de diferentes técnicas, estrategias, métodos... y, por supuesto, de diferentes tecnologías. El aprendizaje de las personas y su situación no es la misma; un mismo foro, un mismo recurso, un mismo ejercicio, una misma actividad son eficaces o ineficaces de un día para otro con uno u otro alumno/a, con uno u otro profesor/a y en diferentes materias (Bartolomé, 2008).

En el bachillerato mixto se implementan diversos tipos de estrategias de enseñanza y aprendizaje, en las cuales se llevan a cabo distintas técnicas y actividades, como las siguientes:

- Autoaprendizaje: estudio individual, actividades individuales, proyectos, ensayos, uso de redes sociales, páginas web y plataformas virtuales.

- Aprendizaje colaborativo: resolución de problemas, exposiciones en equipo, proyectos por equipo, prácticas, visitas guiadas, chats, uso de redes sociales, páginas web y plataformas virtuales.

- Aprendizaje a distancia: recursos didácticos que se alojan en un repositorio digital en la página web del Centro de Educación Continua Abierta y a Distancia de la UABJO (CECAD-UABJO). Entre 


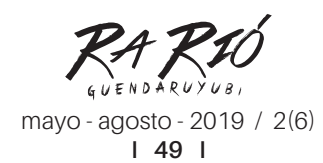

I 49 I

los recursos virtuales se consideran sistemas de correo electrónico personales, webs públicas o privadas, blogs, videos en YouTube, listas ofrecidas por servicios gratuitos, chat vía Skype o Messenger.

- Estrategias de enseñanza: exposiciones por parte del profesorado, clases presenciales en modalidad de asesorías los fines de semana.

- Actividades extracurriculares: son de dos tipos, de formación e integrales. Se llevan a cabo a través de talleres, foros, conferencias, cursos, presenciales y en línea.

En este contexto, el aprendizaje a través de esta modalidad responde de manera efectiva a las necesidades y retos educativos que se tienen en la actualidad a nivel bachillerato, replanteando la manera de interactuar en los procesos de enseñanza y aprendizaje, puesto que se combinan entornos presenciales con medios no presenciales. Es necesario, por lo tanto, determinar y seleccionar los recursos tecnológicos de los que se hará uso para cada necesidad educativa, pues se trata de implementar nuevas formas de generar conocimientos a partir de los contenidos curriculares, los materiales de apoyo y los instrumentos de comunicación a distancia que fundamentan los fines educativos del programa.

Por ello, el bachillerato mixto del CECAD-UABJO propone configurar comunidades de aprendizaje a través de un modelo flexible, en el que un entorno educativo presencial y virtual se integre continua, eficaz y pertinentemente. 


\section{RARIó}

mayo - agosto - 2019 / 2(6)

I 50 I

\section{Referencias}

Pimienta Prieto, J. (2012). Las competencias en la docencia universitaria. México: Pearson.

Ruiz Iglesias, M. (2012). Enseñar en términos de competencia. México: Trillas.

\section{Referencias electrónicas}

Bartolomé-Pina, A. (2008). Entornos de aprendizaje mixto en educación superior. Revista Iberoamericana de Educación a Distancia, 11(1), 15-51. Recuperado de http://revistas. uned.es/index.php/ried/article/view/955

Silva Rodríguez, A., Mendoza Paredes, D. \& Guarneros Reyes, E. (s.f.). Sistema Educativo Mixto, un modelo para la educación del futuro en México. $X$ Congreso Nacional de Investigación Educativa. Área 8. Filosofía, teoría y campo de la educación. Recuperado de http://www.comie.org.mx/congreso/memoriaelectronica/v10/pdf/area_tematica_07/ ponencias/0875-F.pdf

Universidad Estatal a Distancia (UNED). (s.f.). ¿Qué son las estrategias de aprendizaje? Recuperado de https://www.uned.ac.cr/academica/images/ceced/docs/Estaticos/ contenidos.pdf 